\title{
AN IDENTITY WITH DERIVATIONS IN PRIME RINGS
}

\author{
SHULIANG HUANG
}

Received 03 February, 2015

\begin{abstract}
Let $R$ be a prime ring with center $Z(R)$, and $d$ a derivation of $R$. Suppose that $\left(d[x, y]_{k}\right)^{n}-m[x, y]_{k} \in Z(R)$ for all $x, y \in R$, where $m \neq n, k \geq 1$ are fixed integers. Then $d=$ 0 or $R$ satisfies $s_{4}$, the standard identity in four variables. In the case $\left(d[x, y]_{k}\right)^{n}-m[x, y]_{k}=0$ for all $x, y \in R$, then $d=0$ or $R$ is commutative.
\end{abstract}

2010 Mathematics Subject Classification: 16N60; 16W25; 16U80; 16D90

Keywords: prime and semiprime rings, derivations, GPIs

\section{INTRODUCTION}

In all that follows, unless stated otherwise, $R$ will be an associative ring, $Z(R)$ the center of $R, Q$ its Martindale quotient ring and Utumi quotient ring $U$. The center of $Q$, denoted by $C$, is called the extended centroid of $R$ (we refer the reader to [3] for related symbols). For any $x, y \in R$, the symbol $[x, y]$ and $x \circ y$ stand for the commutator $x y-y x$ and anti-commutator $x y+y x$, respectively. Recall that a ring $R$ is prime if for any $a, b \in R, a R b=(0)$ implies $a=0$ or $b=0$ and is semiprime if for any $a \in R, a R a=\{0\}$ implies $a=0$. By a derivation on $R$ we mean an additive mapping $d: R \longrightarrow R$ such that $d(x y)=d(x) y+x d(y)$ holds for all $x, y \in R$. In particular $d$ is called an inner derivation induced by an element $a \in R$, if $d(x)=[a, x]$ for all $x \in R$. For any $x, y \in R$, we set $[x, y]_{1}=[x, y]=x y-y x$, and $[x, y]_{k}=$ $\left[[x, y]_{k-1}, y\right]$, where $k>1$ is an integer. Note that $[x, y]_{k}=\sum_{i=0}^{k}(-x)^{i} y x^{k-i}$ and $d\left([x, y]_{k}\right)=[d(x), y]_{k}+\sum_{i=1}^{k}\left[\left[[x, y]_{i-1}, d(y)\right], y\right]_{k-i}$.

Many results in literature indicate that global structure of a ring $R$ is often lightly connected to the behavior of additive mappings defined on $R$. The first classic result on this topic is due to Divinsky [7] who proved that a simple Artinian ring is commutative if it has a commuting non-identity automorphism. Over the last few decades, a number of authors have investigated the relationship between the commutativity of

The author was supported by the Anhui Provincial Natural Science Foundation (Grant No. 1808085MA14) and the Key University Science Research Project of Anhui Province (Grant No. KJ2018A0433) and Research Project of Chuzhou University (Grant No. zrjz2017005) of P.R. China. 
the ring $R$ and certain specific types of derivations of $R$. In [2], Ashraf and Rehman proved that if $R$ is a prime ring, $I$ a nonzero ideal of $R$ and $d$ is a derivation of $R$ such that $d(x \circ y)=x \circ y$ for all $x, y \in I$, then $R$ is commutative. In [1], Argaç and Inceboz generalized the above result as following: Let $R$ be a prime ring, $I$ a nonzero ideal of $R$ and $n$ a fixed positive integer, if $R$ admits a derivation $d$ with the property $(d(x \circ y))^{n}=x \circ y$ for all $x, y \in I$, then $R$ is commutative. On the other hand, Daif and Bell [6] showed that if in a semiprime ring $R$ there exists a nonzero ideal $I$ of $R$ and a derivation $d$ such that $d([x, y])=[x, y]$ for all $x, y \in I$, then $I \subseteq Z(R)$. In particular, if $I=R$ then $R$ is commutative. Motivated by the above-cited results, our purpose in this article is to obtain some information of the prime ring $R$ involving a central identity $\left(d[x, y]_{k}\right)^{n}-m[x, y]_{k} \in Z(R)$ for all $x, y \in R$, where $m, n, k \geq 1$ are fixed integers.

The standard identity $s_{4}$ in four variables is defined as follows:

$$
s_{4}=\sum(-1)^{\tau} X_{\tau(1)} X_{\tau(2)} X_{\tau(3)} X_{\tau(4)}
$$

where $(-1)^{\tau}$ is the sign of a permutation $\tau$ of the symmetric group of degree 4 . As is well known, prime rings satisfying $s_{4}$ can be characterized by the following:

Fact 1 ([4]). Let $R$ be a prime ring with the extended centroid $C$. Then the following are equivalent:

(1) $\operatorname{dim}_{C} R C \leq 4$;

(2) $R$ satisfies $s_{4}$;

(3) $R$ is commutative or $R$ embeds in $M_{2}(F)$;

(4) $R$ is algebraic of bounded degree 2 over $C$;

(5) $R$ satisfies $\left[\left[x^{2}, y\right],[x, y]\right]$.

\section{Results}

We begin with the following lemmas which are crucial for proving our main results. 
Lemma 1. Let $R=M_{2}(F)$ be the ring of all $2 \times 2$ matrices over a field $F$. If $0 \neq a \in R$ such that

$$
\left([[a, x], y]_{k}+\sum_{i=1}^{k}\left[\left[[x, y]_{i-1},[a, y]\right], y\right]_{k-i}\right)^{n}=m[x, y]_{k}
$$

for all $x \in R$ where $m, n, k \geq 1$ are fixed integers, then $a \in Z(R)$.

Proof. Let $a=\sum_{i, j=0}^{2} a_{i j} e_{i j}$ with $a_{i j} \in F$, where $e_{i j}$ is the usual matrix unit with 1 in $(i, j)$-entry and zero elsewhere. Let $x=e_{12}, y=e_{11}$. Then $m[x, y]_{k}=$ 0 and $\left([[a, x], y]_{k}+\sum_{i=1}^{k}\left[\left[[x, y]_{i-1},[a, y]\right], y\right]_{k-i}\right)^{n}=(-1)^{k n}\left(-e_{12} a+e_{11} a e_{12}\right)^{n}$. By assumption we have $(-1)^{k n}\left(-e_{12} a+e_{11} a e_{12}\right)^{n}=0$. Right multiplying by $e_{12}$, it yields that $(-1)^{(k+1) n} a_{21}^{n} e_{12}=0$, which implies $a_{21}=0$. Similarly, we have $a_{12}=0$. Thus $a$ must be a diagonal matrix. Now set $a=\sum_{t} a_{t t} e_{t t}$, with $a_{t t} \in F$. Let $\varphi$ be the inner automorphism of $R$ given by $\varphi(x)=\left(1+e_{i j}\right) a\left(1-e_{i j}\right)$. Thus $\left(\left[\left[a^{\varphi}, x\right], y\right]_{k}+\sum_{i=1}^{k}\left[\left[[x, y]_{i-1},\left[a^{\varphi}, y\right]\right], y\right]_{k-i}\right)^{n}=m[x, y]_{k}$ for all $x, y \in R$. By above argument,

$$
a^{\varphi}=\left(1+e_{i j}\right) a\left(1-e_{i j}\right)=\Sigma_{i=1}^{k} a_{i i} e_{i i}+\left(a_{j j}-a_{i i}\right) e_{i j}
$$

must be diagonal. Therefore $a_{j j}=a_{i i}$ and so $a \in Z(R)$.

Lemma 2. Let $R$ be a non-commutative prime ring with center $Z(R)$. If $0 \neq a \in R$ such that

$$
\left([[a, x], y]_{k}+\sum_{i=1}^{k}\left[\left[[x, y]_{i-1},[a, y]\right], y\right]_{k-i}\right)^{n}=m[x, y]_{k}
$$

for all $x, y \in R$, where $n, m, k \geq 1$ are fixed integers, then $a \in Z(R)$.

Proof. By assumption, $R$ satisfies the generalized polynomial identity

$$
p(x, y)=\left([[a, x], y]_{k}+\sum_{i=1}^{k}\left[\left[[x, y]_{i-1},[a, y]\right], y\right]_{k-i}\right)^{n}-m[x, y]_{k} .
$$

By Chuang [5], this generalized polynomial identity (GPI) is also satisfied by $U$. If $a \notin C$ then $p(x, y)=0$ is a nontrivial (GPI) for $U$. In case $C$ is infinite, we have $p(x, y)=0$ for all $x, y \in U \otimes_{C} \bar{C}$ where $\bar{C}$ is the algebraic closure of $C$. Since both $U$ and $U \otimes_{C} \bar{C}$ are prime and centrally closed [8], we may replace $R$ by $U$ or $U \otimes_{C} \bar{C}$ according to $C$ finite or infinite. Thus we may assume that $R$ is centrally closed over $C$ which is either finite or algebraically closed and $p(x, y)=0$ for all $x, y \in R$. By Martindale's theorem [13], $R$ is then a primitive ring having nonzero $\operatorname{soc}(R)$ with $C$ as the associated division ring. Hence by Jacobson's theorem [9], $R$ is isomorphic to a dense ring of linear transformations of a vector space $V$ over $C$. If $\operatorname{dim}_{C} V=k$, then the density of $R$ on $V$ implies that $R \cong M_{k}(C)$, where $k=\operatorname{dim}_{C} V$. Since $R$ is noncommutative, $k \geq 2$. If $\operatorname{dim}_{C} V=2$, then by Lemma 1 we 
have $a \in Z(R)$. Now suppose that $\operatorname{dim}_{C} V \geq 3$. We want to show that for any $v \in V$, $v$ and $a v$ are linearly $C$-dependent. Suppose on contrary that $v$ and $a v$ are linearly $C$-independent for some $v \in V$. Since $\operatorname{dim}_{C} V \geq 3$, there exists $w \in V$ such that $\{v, a v, w\}$ are linearly $C$-independent set of vectors. By the density of $R$ on $V$, there exist $x, y \in R$ such that $x v=v, x w=v+w, x a v=w ; y v=v, y w=0, y a v=v$. Then $0=p(x, y) v=(-1)^{k n} v$, a contradiction. Therefore $v, a v$ are linearly $C$ dependent for any $v \in V$. Hence we can write $a v=v \alpha_{v}$ for all $v \in V$ and $\alpha_{v} \in C$. Then by a standard argument, it is very easy to prove that $\alpha_{v}$ is independent of the choice of $v \in V$. In fact, Since $\operatorname{dim}_{C} V \geq 3$, then there exist $u, v, w$ which are linearly independent, and so $\alpha_{u}, \alpha_{v}, \alpha_{w} \in C$ such that $a u=u \alpha_{u}, a v=v \alpha_{v}, a w=$ $w \alpha_{w}$, that is $a(u+v+w)=u \alpha_{u}+v \alpha_{v}+w \alpha_{w}$. Moreover $a(u+v+w)=(u+$ $v+w) \alpha_{u+v+w}$ for some $\alpha_{u+v+w} \in C$. Then $0=\left(\alpha_{u+v+w}-\alpha_{u}\right) u+\left(\alpha_{u+v+w}-\right.$ $\left.\alpha_{v}\right) v+\left(\alpha_{u+v+w}-\alpha_{w}\right) w$ and hence $\alpha_{u}=\alpha_{v}=\alpha_{w}=\alpha_{u+v+w}$, that is $\alpha$ does not depend on the choice of $v$. Thus we can write $a v=v \alpha$ for all $v \in V$ and $\alpha \in C$ fixed. Now, let $r \in R, v \in V$. Since $a v=v \alpha$, we have $[a, r] v=(a r) v-(r a) v=$ $a(r v)-r(a v)=(r v) \alpha-r(v \alpha)=0$, that is $[a, r] V=0$. Hence $[a, r]=0$ for all $r \in R$, implying $a \in Z(R)$.

Theorem 1. Let $R$ be a prime ring and $d$ a derivation of $R$. Suppose that $\left(d[x, y]_{k}\right)^{n}=m[x, y]_{k}$ for all $x, y \in R$, where $m \neq n, k \geq 1$ are fixed integers. Then $d=0$ or $R$ is commutative.

Proof. Using the identity $d\left([x, y]_{k}\right)=[d(x), y]_{k}+\sum_{i=1}^{k}\left[\left[[x, y]_{i-1}, d(y)\right], y\right]_{k-i}$ and the hypothesis, we have

$$
\left([d(x), y]_{k}+\sum_{i=1}^{k}\left[\left[[x, y]_{i-1}, d(y)\right], y\right]_{k-i}\right)^{n}=m[x, y]_{k}
$$

for all $x, y \in R$. Assume first that $d$ is $Q$-inner, that is, $d(x)=[a, x]$ for all $x \in Q$, where $a$ is a non-central element in $Q$. Then

$$
\left([[a, x], y]_{k}+\sum_{i=1}^{k}\left[\left[[x, y]_{i-1},[a, y]\right], y\right]_{k-i}\right)^{n}=m[x, y]_{k}
$$

for all $x, y \in U$. Thus by Lemma 2, $a \in Z(R)$ that gives $d=0$.

Assume next that $d$ is $Q$-outer. Applying Kharchenko's theorem [10], we get

$\left([v, y]_{k}+\sum_{i=1}^{k}\left[\left[[x, y]_{i-1}, u\right], y\right]_{k-i}\right)^{n}=m[x, y]_{k}$ for all $x, y, u, v \in U$. In particular, for $u=0$ and $v=x$ we have $\left([x, y]_{k}\right)^{n}=m[x, y]_{k}$, for all $x, y \in U$. Note that, this is a polynomial identity and hence there exists a field $F$ such that $R \subseteq M_{t}(F)$, the ring of $t \times t$ matrices over a field $F$, where $t \geq 1$. By Chuang [5], this generalized polynomial identity (GPI) is also satisfied by $R$ as well. Moreover, $R$ and $M_{t}(F)$ satisfy the same polynomial identity [11, Lemma 1], that is, $\left([x, y]_{k}\right)^{n}=m[x, y]_{k}$ for all $x, y \in M_{t}(F)$. But by choosing $x=e_{12}, y=e_{11}$, we get $0=\left([x, y]_{k}\right)^{n}-m[x, y]_{k}=$ $(-1)^{k}\left(e_{12}^{n}-m e_{12}\right)$. This is a contradiction, ending the proof. 
Lemma 3. Let $R=M_{t}(F)$ be the ring of all $t \times t$ matrices over a field $F$ with $t \geq 3$. If $0 \neq a \in R$ and $m, n, k \geq 1$ are fixed integers, such that ([[a,x],y $]_{k}+$ $\left.\sum_{i=1}^{k}\left[\left[[x, y]_{i-1},[a, y]\right], y\right]_{k-i}\right)^{n}-m[x, y]_{k} \in F \cdot I_{t}$, for all $x, y \in R$, then $a \in F \cdot I_{t}$.

Proof. We are given that

$$
\left[\left([[a, x], y]_{k}+\sum_{i=1}^{k}\left[\left[[x, y]_{i-1},[a, y]\right], y\right]_{k-i}\right)^{n}-m[x, y]_{k}, z\right]=0
$$

for all $x, y, z \in R$. Let $a=\left(a_{i j}\right)_{t \times t}$. By choosing $x=e_{i j}, y=e_{i i}$ and $z=e_{i k}$ for any $i \neq j \neq k$, we have

$$
\left[(-1)^{k n}\left[a, e_{i j}\right]^{n}-(-1)^{k} m e_{i j}, e_{i k}\right]=(-1)^{k n}\left(\left(e_{i j} a\right)^{n} e_{i k}-e_{i k}\left(a e_{i j}\right)^{n}\right)=0 .
$$

Thus $a_{i j}=0$, and so $a$ is a diagonal matrix. Using the same technique in Lemma 1, we get $a \in F \cdot I_{t}$, proving the lemma.

Theorem 2. Let $R$ be a prime ring with center $Z(R)$, and $d$ a derivation of $R$. Suppose that $\left(d[x, y]_{k}\right)^{n}-m[x, y]_{k} \in Z(R)$ for all $x, y \in R$, where $m \neq n, k \geq 1$ are fixed integers. Then $d=0$ or $R$ satisfies $s_{4}$, the standard identity in four variables.

Proof. By assumption $R$ satisfies the generalized differential identity

$0=\left[\left(d[x, y]_{k}\right)^{n}-m[x, y]_{k}, w\right]=\left[[d(x), y]_{k}+\sum_{i=1}^{k}\left[\left[[x, y]_{i-1}, d(y)\right], y\right]_{k-i}-m[x, y]_{k}, w\right]$

for $x, y, w \in R$. By Lee [12], $R$ and $U$ satisfy the same differential identities we may assume that above identity is also satisfied by $U$. Now we consider the following two cases:

Case 1. Suppose that $d$ is a $Q$-outer derivation. Then Kharchenko's theorem [10], we have

$$
\left[\left([v, y]_{k}+\sum_{i=1}^{k}\left[\left[[x, y]_{i-1}, u\right], y\right]_{k-i}\right)^{n}-m[x, y]_{k}, w\right]=0,
$$

for all $x, y, u, v, w \in U$. This is a polynomial identity and hence there exists a field $F$ such that $U \subseteq M_{t}(F)$ with $t>1$ and $U, M_{t}(F)$ satisfy the same polynomial identity [11]. If $t \geq 3$ then by choosing $w=e_{13}, v=x=e_{12}, y=e_{11}, u=0$, we get $0=\left[\left([v, y]_{k}+\sum_{i=1}^{k}\left[\left[[x, y]_{i-1}, u\right], y\right]_{k-i}\right)^{n}-m[x, y]_{k}, w\right]=(-1)^{k} e_{13}$. This is a contradiction. Thus $t=2$ and so $R$ satisfies $s_{4}$ by Fact 1 . 
Case 2. Suppose that $d$ is a $Q$-inner derivation. In this case there exists $a \in Q$ such that $d(x)=[a, x]$ for all $x \in R$. Then we have

$$
\left[\left([[a, x], y]_{k}+\sum_{i=1}^{k}\left[\left[[x, y]_{i-1},[a, y]\right], y\right]_{k-i}\right)^{n}-m[x, y]_{k}, w\right]=0,
$$

for all $x, y, w \in R$. By localizing $R$ at $Z(R)$ it follows that

$$
\left([[a, x], y]_{k}+\sum_{i=1}^{k}\left[\left[[x, y]_{i-1},[a, y]\right], y\right]_{k-i}\right)^{n}-m[x, y]_{k} \in Z\left(R_{Z}\right),
$$

for all $x, y \in R_{Z}$. Since $R$ and $R_{Z}$ satisfy the same polynomial identities, in order to prove that $R$ satisfies $s_{4}$, we may assume that $R$ is simple with 1 . Hence, $\left([[a, x], y]_{k}+\sum_{i=1}^{k}\left[\left[[x, y]_{i-1},[a, y]\right], y\right]_{k-i}\right)^{n}-m[x, y]_{k} \in Z(R)$ for all $x, y \in R$. Therefore $R$ satisfies a generalized polynomial identity and it is simple with 1 , which implies that $Q=R C=R$ and $R$ has a minimal right ideal, whose commuting ring $D$ is a division ring which is finite dimensional over $Z(R)$. However, since $R$ is a simple ring with $1, R$ must be Artinian, that is, $R=D_{s}$, the $s \times s$ matrices over $D$, for some $s \geq 1$. By [11] there exists a field $F$ such that $R \subseteq M_{t}(F)$, the ring of $t \times t$ matrices over field $F$, with $t>1$, and

$$
\left([[a, x], y]_{k}+\sum_{i=1}^{k}\left[\left[[x, y]_{i-1},[a, y]\right], y\right]_{k-i}\right)^{n}-m[x, y]_{k} \in Z\left(M_{t}(F)\right)=F \cdot I_{t},
$$

for all $x, y \in M_{t}(F)$. If $t \geq 3$, then by Lemma 3, $a \in F \cdot I_{t}$ and so $d=0$. If $t=2$, then $R$ satisfies $s_{4}$.

\section{ACKNOWLEDGEMENT}

The author would like to thank the referees for their helpful comments.

\section{REFERENCES}

[1] N. Argaç and H. G. Inceboz, "Derivations of prime and semiprime rings," SIAM J. Korean Math. Soc., vol. 46, no. 5, pp. 997-1005, 2009, doi: 10.4134/JKMS.2009.46.5.997.

[2] M. Ashraf and N. Rehman, "On commutativity of rings with derivations," SIAM Results Math., vol. 42, no. 1-2, pp. 3-8, 2002, doi: https://doi.org/10.1007/BF03323547.

[3] K. I. Beidar, W. S. Martindale III, and A. V. Mikhalev, Rings with generalized identities. New York: Marcel Dekker, 1996. doi: 10.1007/978-1-4614-6946-9.

[4] M. Bresar, "Commuting traces of biadditive mappings, commutativity-preserving mappings and Lie mappings," SIAM Tran. Amer. Math. Soc., vol. 335, no. 2, pp. 525-546, 1993, doi: $10.2307 / 2154392$.

[5] C. L. Chuang, "GPIs having coefficients in Utumi quotient rings," SIAM Proc. Amer. Math. Soc., vol. 103, no. 3, pp. 723-728, 1988, doi: 10.2307/2046841.

[6] M. N. Daif and H. E. Bell, "GPIs Remarks on derivations on semiprime rings," SIAM Int. J. Math. Math. Sci., vol. 15, no. 1, pp. 205-206, 1992, doi: 10.1155/S0161171292000255. 
[7] N. Divinsky, "On commuting automorphisms of rings," SIAM Proc. Trans. R. Soc. Canada III (3), vol. 49, no. 3, pp. 19-22, 1955.

[8] T. S. Erickson, W. S. Martindale, and J. M. Osborn, "Prime nonassociative algebras," SIAM Pacific J. Math., vol. 60, no. 1, pp. 49-63, 1975, doi: 10.2140/pjm.1975.60.49.

[9] N. Jacobson, Structure of rings. New York: Marcel Dekker, 1964. doi: 10.1090/s0002-99041957-10071-8.

[10] V. K. Kharchenko, "Differential identity of prime rings," SIAM Algebra and Logic, vol. 17, no. 2, pp. 155-168, 1978, doi: https://doi.org/10.1007/BF01670115.

[11] C. Lanski, "An engle condition with derivation," SIAM Proc. Amer. Math. Soc., vol. 183, no. 3, pp. 731-734, 1993, doi: 10.2307/2160113.

[12] T. K. Lee, "Semiprime rings with differential identities," SIAM Bull. Inst. Math. Acad. Sinica., vol. 20, no. 1, pp. 27-38, 1992.

[13] W. S. Martindale 3rd, "Prime rings satistying a generalized polynomial identity," SIAM J. Algebra, vol. 12, no. 4, pp. 576-584, 1969, doi: https://doi.org/10.1016/0021-8693(69)90029-5.

Author's address

\section{Shuliang Huang}

Chuzhou University, School of Mathematics and Finance, 239000 Chuzhou P. R. China

E-mail address: shulianghuang@163.com 